\title{
In-hospital outcomes after switching from a bivalirudin-first strategy to an unfractionated heparin-first strategy for percutaneous coronary interventions
}

\author{
Rahul V. Jaswaney ${ }^{1}$, Melissa C. Caughey ${ }^{2}$, Christopher End ${ }^{2}$, Patricia Sudar ${ }^{2}$, Michael Yeung ${ }^{2}$, Prashant \\ Kaul $^{2,3}$, Joseph S. Rossi ${ }^{2}$, George A. Stouffer ${ }^{2}$, John P. Vavalle ${ }^{2}$ \\ ${ }^{1}$ School of Medicine, ${ }^{2}$ Division of Cardiology, Department of Medicine, University of North Carolina at Chapel Hill, Chapel Hill, NC, USA; \\ ${ }^{3}$ Piedmont Heart Institute, Atlanta, GA, USA \\ Contributions: (I) Conception and design: RV Jaswaney, JP Vavalle; (II) Administrative support: P Sudar; (III) Provision of study material or patients: \\ JP Vavalle, P Kaul, M Yeung, JS Rossi, GA Stouffer; (IV) Collection and assembly of data: RV Jaswaney, MC Caughey, P Sudar; (V) Data analysis and \\ interpretation: RV Jaswaney, MC Caughey, JP Vavalle; (VI) Manuscript writing: All authors; (VII) Final approval of manuscript: All authors. \\ Correspondence to: John P. Vavalle, MD, MHS, FACC. School of Medicine, University of North Carolina at Chapel Hill, 160 Dental Circle, CB\# \\ 7075, Chapel Hill, NC 27599-7075, USA. Email: john_vavalle@med.unc.edu.
}

\begin{abstract}
Background: The optimal anticoagulation strategy for percutaneous coronary interventions (PCIs) remains debated. We report outcomes after switching from a bivalirudin-first to an unfractionated heparin (UFH)-first strategy for PCIs in a large academic center.

Methods: Patients undergoing PCI from June $1^{\text {st }} 2013-$ May $31^{\text {st }}, 2015$ were identified through the National Cardiovascular Data Registry (NCDR), and divided into the "bivalirudin era" (June 2013-July 2014) and the "UFH era" (October 2014-May 2015). Bleeding outcomes were compared using multivariable logistic regression adjusted for potential confounders.

Results: A total of 1,145 patients were identified (bivalirudin era $=752$, UFH era $=393$ ). Radial access for PCI increased over time, and was lower in the bivalirudin era (26\% vs. $34 \%, \mathrm{P}<0.05)$. There were 32 major bleeds $(4.3 \%)$ in the bivalirudin era and 29 major bleeds $(7.4 \%)$ in the UFH era $(\mathrm{P}=0.03)$, with the majority being hemoglobin drops ( $\geq 3 \mathrm{~g} / \mathrm{dL}$ ) without overt clinical bleeding $(85.7 \%$ of bleeds in the bivalirudin era and $86.2 \%$ of bleeds in the UFH era). After adjustments for other common major causes of bleeding, bivalirudin was associated with $78 \%$ lower odds of bleeding (OR =0.22; 95\% CI: 0.05-0.91).

Conclusions: An increase in major bleeding events occurred after switching to an UFH-first strategy, primarily associated with hemoglobin drop ( $\geq 3 \mathrm{~g} / \mathrm{dL})$ without overt clinical bleeding. Major overt bleeding was rare $(0.3 \%)$ and similar in both groups. These results suggest a UFH-first strategy for PCI may have a role in patients with low bleeding risk.
\end{abstract}

Keywords: Bivalirudin; bleeding; percutaneous coronary intervention (PCI); unfractionated heparin (UFH)

Submitted Sep 15, 2017. Accepted for publication Oct 17, 2017.

doi: $10.21037 /$ cdt.2017.10.16

View this article at: http://dx.doi.org/10.21037/cdt.2017.10.16

\section{Introduction}

Since the initial days of balloon angioplasty, anticoagulants agents such as unfractionated heparin (UFH) or bivalirudin, either alone or in combination with a glycoprotein IIb/ IIIa inhibitor (GPI), have been used during percutaneous coronary intervention (PCI) (1). Major adverse bleeding complications from PCI have been shown to have a strong association with poor prognosis including higher 30-day mortality rates $(2,3)$. Other studies have shown a strong, dose-dependent relationship between major bleeding and mortality at 1 year (4).

Studies of bivalirudin have shown lower bleeding risk 
when compared to a heparin (either UFH or low molecular weight heparin) + routine GPI strategy, but there are conflicting results when bivalirudin is compared to heparin + provisional GPI strategy. In 2003, the Randomized Evaluation in PCI Linking Angiomax to Reduced Clinical Events (REPLACE-2) compared bivalirudin + provisional GPI use vs. UFH + routine GPI use during elective and urgent PCI. There was no difference between the two groups in 30-day death, myocardial infarction (MI), or need for urgent revascularization but the rate of in-hospital major bleeding was reduced in the bivalirudin group (5). The 2007 Acute Catheterization and Urgent Intervention Triage strategY (ACUITY) trial and 2008 Harmonizing Outcomes with RevasculariZatiONs and Stents in Acute Myocardial Infarction (HORIZONS-AMI), reported similar results with regard to reduction in major bleeding in patients treated with bivalirudin alone, as compared the UFH + GPI, without any difference in ischemic endpoints in patients undergoing PCI for treatment of acute coronary syndromes $(2,6,7)$. A subgroup analysis of ACUITY comparing UFH + routine GPI use and bivalirudin + routine GPI use found similar rates of major bleeding (7). Analyses of these studies suggested that the majority of the bleeding complications during PCI can be attributed to GPI use. In current practice, GPI use has declined due to the availability of more potent oral P2Y12 inhibitors and is typically reserved as a "bailout" strategy for cases with high thrombus burden (7).

The REPLACE-2, ACUITY, and HORIZONS studies were conducted in an era where GPI use and femoral access were common. The RadIal $v s$. femorAL (RIVAL) multicenter randomized controlled trial demonstrated that radial access significantly reduced major vascular complications, such as large hematomas and pseudoaneurysms needing closure compared to femoral access (8). The UK-based, single center randomized control trial, How Effect Are Antithrombotic Therapies trial (HEAT-PPCI), randomly assigned patients to either UFH or bivalirudin treatment with predominantly radial access and provisional GPI use (9) and found no significant difference in bleeding outcomes between UFH monotherapy and bivalirudin monotherapy (9). Recently, the Bivalirudin in Acute Myocardial Infarction vs. UFH and GPI plus UFH Trial (BRIGHT) and Minimizing Adverse Hemorrhagic Events by Transradial Access Site and Systemic Implementation of AngioX (MATRIX) were also conducted using modern techniques in PCI. These trials found conflicting results with the BRIGHT trial demonstrating bivalirudin significantly reduced the primary endpoint of a composite of major adverse cardiac events, cerebral events, or any bleeding without any difference in acute in-stent thrombosis at 1-year. MATRIX demonstrated no difference in a composite of death from any cause, MI or stroke between the groups but bivalirudin was associated with a significant reduction in major bleeding (BARC 3 or 5) and fatal bleeding with a borderline increase in acute instent thrombosis.

In response to data showing reduced bleeding risk with radial access, the availability of newer and more potent P2Y12 inhibitors, and the significantly higher cost of bivalirudin compared to UFH, the University of North Carolina Catheterization Laboratory made a concerted effort in June 2014 to switch from bivalirudin to UFH for the majority of PCI. This study aimed to analyze the outcomes approximately 1 year after the switch in preferred anticoagulant.

\section{Methods}

This was a cross-sectional analysis of all patients who underwent PCI for any reason at the UNC Cardiac Catheterization Laboratory between June 2013 and May 2015. Patients were identified through the National Cardiovascular Data Registry (NCDR) as having undergone PCI if they had "percutaneous coronary intervention (e.g., stent, atherectomy, or thrombectomy catheter) into a native coronary artery or coronary artery bypass graft (CABG) for the purpose of mechanical coronary revascularization." Our analysis was limited to the first occurring PCI performed per patient during the study observation period. The interventional cardiologists working in the laboratory agreed to switch from bivalirudin to UFH as the firstchoice anticoagulation during PCI in June 2014, with the transition occurring in August and September 2014. For the purposes of this study, patients were assigned to one of two groups based on date of catheterization: June 2013-July 2014 representing bivalirudin as first choice anticoagulation (i.e., "bivalirudin era") and October 2014-May 2015 representing UFH as first choice anticoagulation (i.e., "UFH era”). The transition period of August-September 2014 was eliminated from analysis. This study was approved by the institutional review board of the University of North Carolina at Chapel Hill.

Patient demographics (age, sex, weight, and race) and clinical characteristics were abstracted from the medical record at the time of catheterization. Cardiovascular risk 
factors such recent smoking (within 1 year), diabetes mellitus, hypertension, dyslipidemia, family history of premature coronary artery disease, history of prior MI, prior PCI, or prior CABG were recorded, as was coronary artery disease presentation, arterial access site for catheterization, target vessel for revascularization, and cardiogenic shock. Renal disease was derived from the preprocedural serum creatinine, using the CKD-Epi formula (GFR $<60 \mathrm{~mL} / \mathrm{min} / 1.73 \mathrm{~m}^{2}$ ).

Procedural medications were defined as any medication received by the patient 24 hours prior to and during the PCI procedure. Aspirin use, P2Y12 inhibitor use (clopidogrel, prasugrel, or ticagrelor), anticoagulant use (bivalirudin or UFH), and GPI use were recorded. Some patients may have been initially started on UFH drip prior to catheterization depending on presentation acuity. From there, patients will have received a loading dose of $\mathrm{P} 2 \mathrm{Y} 12$ inhibitor and either UFH or bivalirudin would be used for the PCI. This study was approved by the UNC institutional review board (IRB 15-0537).

\section{Patient outcomes}

Patient outcomes focused around the NCDR defined major bleeding events occurring within 72 hours after PCI. The 72-hour bleeding endpoint was chosen as the primary endpoint of this study as it proved the most reliably reported and verifiable endpoint. Other endpoints, such as 30-day mortality, 30-day rethrombosis, or 12-month restenosis proved difficult to verify and inconsistently reported. Multiple studies have demonstrated that major bleeding events have demonstrated a strong association between periprocedural bleeding loss and long-term outcomes for patients undergoing PCI. The NCDR defines a major bleeding event as a bleed at access site, hemoglobin drop of $\geq 3 \mathrm{~g} / \mathrm{dL}$, hematoma at access site, retroperitoneal (RP) bleed, gastrointestinal (GI) bleeding, and genitalurinary $(\mathrm{GU})$ bleeding. Bleed at access site was defined as unexplained drop in hemoglobin $\geq 3 \mathrm{~g} / \mathrm{dL}$, or drop in hemoglobin requiring transfusion of whole blood or packed red blood cells, attributed to intra-procedural blood loss. Hematoma at access site was defined as a palpable or imaged hematoma experienced at the percutaneous entry site of any size, whereas GI, GU, and $\mathrm{RP}$ bleeding were defined as bleeding noted on exam, imaging, or requiring intervention in their respective body compartment. Hemoglobin drop was a common cause of major bleed event and was separately tabulated.

\section{Statistical methods}

Baseline characteristics and clinical outcomes were analyzed, contrasting the bivalirudin era to the UFH era. Continuous variables were assessed for normality, and compared using two sample $t$-tests or Wilcoxon rank sums tests, as appropriate. Categorical variables were compared using a $\chi^{2}$ test. Categorical variables with expected cell counts $<5$ were analyzed by Fisher's exact test. A P value $<0.05$ was considered significant. The odds of major bleeding during the bivalirudin era $v s$. the UFH era were analyzed using multivariable logistic regression. Models accounted for misclassified era-specific anticoagulants (bivalirudin use during the "UFH era" or UFH use during the "bivalirudin era"), other anticoagulants (aspirin, P2Y12 inhibitor, and GPI) as well as demographics (age, race, and sex), diabetes mellitus, CKD, vascular access site, STEMI presentation, and cardiogenic shock. A univariate model was performed to determine the effect of variables such as: age, female, smoking status, hypertension, diabetes, chronic kidney disease, MI, femoral access, cardiogenic shock, bivalirudin use, UFH use, and GPI use. All statistical analyses were carried out using SAS 9.4 (SAS Institute; Cary, NC, USA).

\section{Results}

A total of 1,565 PCIs occurred between June $1^{\text {st }} 2013$ and May $31^{\text {st }} 2015$. There were 134 PCIs that occurred in the transition months that were eliminated from the study and 234 PCIs were eliminated due to either repeat catheterizations or lack of laboratory data to determine bleeding event. The remaining 1,145 PCIs were separated into the two eras based on time, 752 patients occurred in the bivalirudin era (between June 2013 and July 2014) and 393 PCIs occurred in the UFH era (between October 2014 and May 2015). Bivalirudin was used in $88 \%$ of cases during the bivalirudin era and UFH was used in $98 \%$ of cases during the UFH era (Figure 1).

\section{Baseline demographics and presenting symptoms}

Baseline demographic features, were well matched (Table 1) with an average age of 64 years and approximately $70 \%$ of patients being male in both groups. Racial demographics were generally well matched between groups. Patient medical history and CAD risk factors were also matched well, with similar rates of diabetes mellitus, smoking history, hypertension, renal disease and cardiogenic shock (Table 1). 


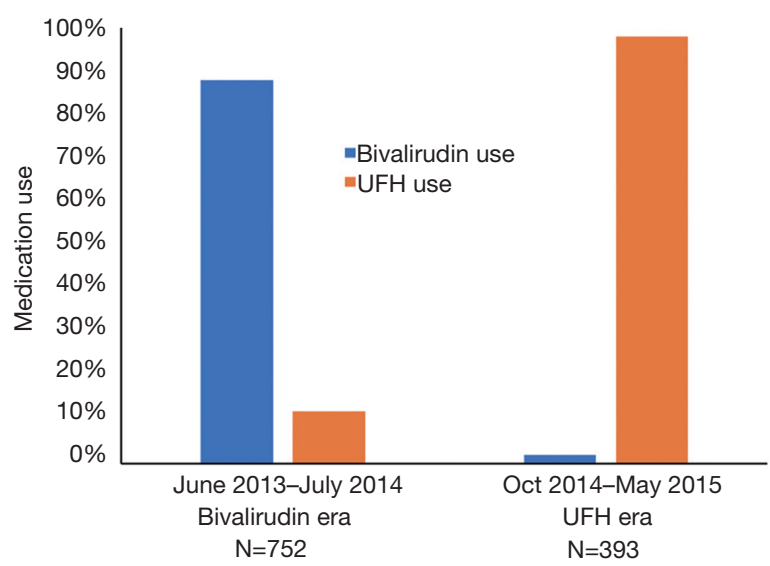

Figure 1 Distributions of bivalirudin and UFH use after switching from a bivalirudin-first to a UFH-first strategy. Distributions of bivalirudin use and UFH use during the bivalirudin era (June 2013-July 2014) and UFH era (October 2014-May 2015). UFH, unfractionated heparin.
There was a slightly higher rate of prior revascularization (53\% vs. $44 \%, \mathrm{P}=0.006)$ particularly with prior PCI $(43 \%$ vs. $35 \%$ ) in the bivalirudin era as compared to the UFH era. Family history of CAD was also lower in UFH era (13\% vs. $25 \%, \mathrm{P}=0.006)$. CAD presentation was similar in both groups (Table 2) with a slightly lower rate of left circumflex as a target vessel in the UFH era.

Aspirin use was near universal throughout the study period of June 2013 to May 2015 (Table 2). Dual antiplatelet use slightly shifted away from clopidogrel (73\% to $65 \%, \mathrm{P}=0.01)$ in the bivalirudin era and toward prasugrel ( $24 \%$ to $30 \%, \mathrm{P}=0.01)$ and ticagrelor ( $1 \%$ to $3 \%, \mathrm{P}=0.002)$ in the UFH era (Table 2). UNC uses a genotyping strategy to determine DAPT in a majority of patients (10). GPI use was low in both groups ( $5 \% v s .6 \%, \mathrm{P}=0.2)$. A sharp downward shift in bivalirudin use was observed during the transition period of August and September 2014 (Figure 2).

Table 1 Patient characteristics at baseline

\begin{tabular}{lccc}
\hline Characteristic & Bivalirudin era June 2013-July 2014 (N=752) & UFH era Oct 2014-May 2015 (N=393) & P value \\
\hline Age (years) & $64 \pm 12$ & $64 \pm 12$ & 0.6000 \\
Male [\%] & $525[70]$ & $269[68]$ & 0.7000 \\
Weight (kg) & $89.1 \pm 21.8$ & $89.6 \pm 21.0$ & 0.6000 \\
White [\%] & $537[71]$ & $277[70]$ & - \\
History and risk factors [\%] & & & 0.8000 \\
Diabetes & $389[43]$ & $237[44]$ & $0.0400^{*}$ \\
Current smoker & $236[26]$ & $115[21]$ & 0.5000 \\
HTN & $771[86]$ & $455[85]$ & $0.0090^{*}$ \\
Hypercholesterolemia & $690[77]$ & $380[71]$ & $<0.0001^{*}$ \\
Family history of CAD & $234[26]$ & $74[13]$ & 0.2000 \\
Prior Ml & $316[35]$ & $170[31]$ & $0.0100^{*}$ \\
Prior PCl & $419[47]$ & $214[39]$ & $<0.0001^{*}$ \\
Prior CABG & $189[21]$ & $189[18]$ & $0.0005^{*}$ \\
CKD (GFR <60) & $251[28]$ & $106[20]$ & 0.7000 \\
Cardiogenic shock & $34[4]$ & $18[3]$ & \\
\hline
\end{tabular}

*, significance to $\mathrm{P}<0.05$. HTN, hypertension; CAD, coronary artery disease; MI, myocardial infarction; CABG, coronary artery bypass graft; CKD, chronic kidney disease; UFH, unfractionated heparin. 
Table 2 Procedure characteristics

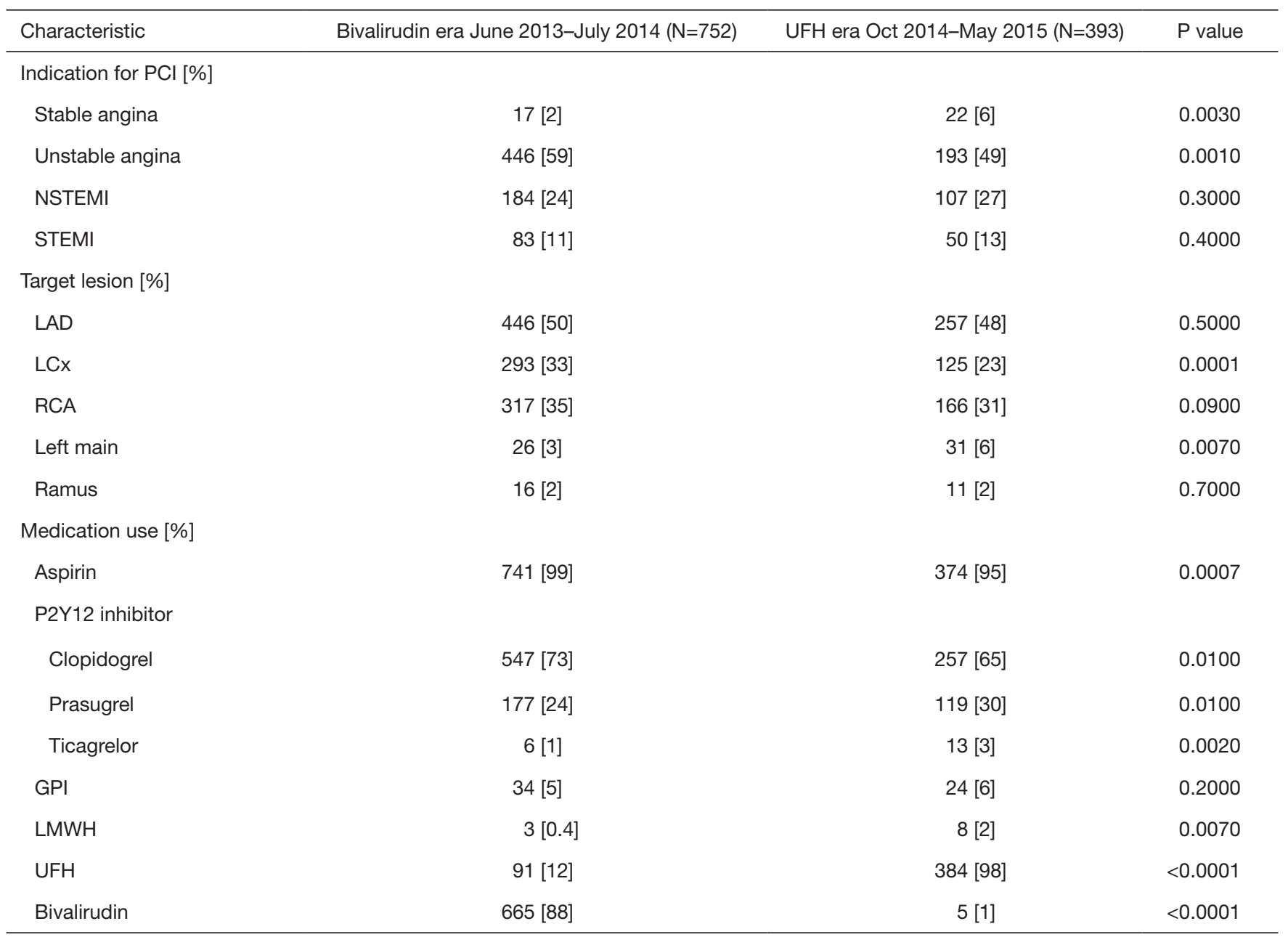

Target lesion was defined as coronary artery stenosis in which revascularization was attempted. Procedures in which multiple coronary interventions were attempted are represented as individual target lesions. NSTEMI, non-ST elevation myocardial infarction; STEMI, ST elevation myocardial infarction; LAD, left anterior descending artery; LCx, left circumflex artery; RCA, right coronary artery; LMWH, low molecular weight heparin; GPI, glycoprotein Ilb/Illa inhibitor; UFH, unfractionated heparin.

A gradual shift in PCI access away from femoral access was observed as femoral access declined from $74 \%$ to $64 \%$ (Figure 2); there was a statistically significant upward trend in radial use $(\mathrm{r}=0.11, \mathrm{P}<0.0001)$.

\section{Patient outcomes}

There were 32 (4.2\%) major bleeds within 72 hours of PCI identified in the bivalirudin era of June 2013-July 2014 and 29 bleeds (7.4\%) identified in UFH era of October 2014-May 2015 with an absolute difference of 3.2\% between the two groups (Table 3, Figure 3).

The majority of major bleeding events were due to a decline in hemoglobin values of at least $3 \mathrm{~g} / \mathrm{dL}$ without other overt sings of bleeding [ 28 of 32 major bleeds in the bivalirudin era $(87.5 \%)$ and 25 of 29 major bleeds in the UFH era $(86.2 \%)]$. In the both the bivalirudin era and UFH era, RP, GU, and GI bleeds were not significantly different, though these events were exceedingly rare making it difficult to compare the two groups accurately. Primary anticoagulant with each bleed demonstrated 28 major bleeds with bivalirudin use $v s .4$ bleeds with UFH use in the bivalirudin era and 28 bleeds with UFH and 1 bleed with bivalirudin during the UFH era. GPI was heavily represented among bleeding events in both eras, with 12 bleeding events in the bivalirudin era and 9 bleeding 


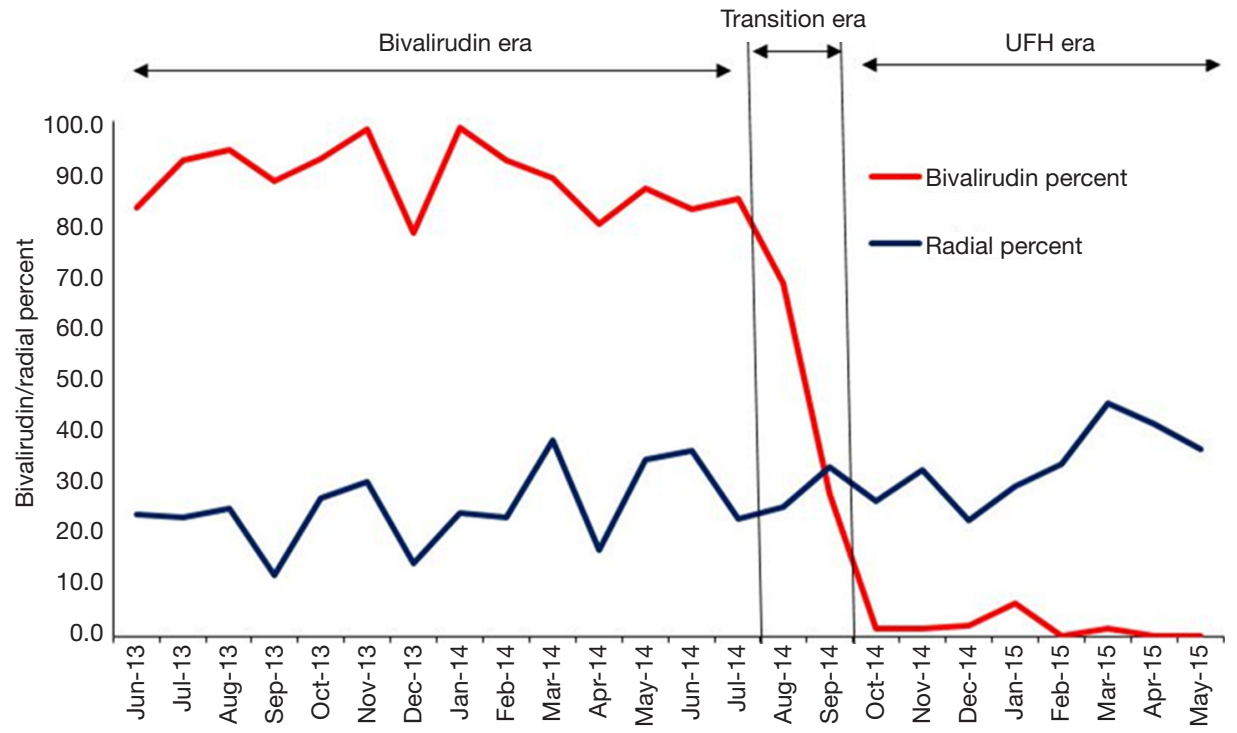

Figure 2 Bivalirudin use and radial access use over time. The use of bivalirudin and radial access plotted over the course of 2 years of this study. Two "eras" were determined: the bivalirudin era (June 2013-July 2014) and the UFH era (October 2014-May 2015). The transition period was from August 2014 to September 2014. There was a statistically significant upward trend in radial access use ( $\mathrm{r}=0.11$, $\mathrm{P}<0.0001$ ) by Pearson correlation. UFH, unfractionated heparin.

Table 3 NCDR-defined major bleeding events by category

\begin{tabular}{|c|c|c|c|}
\hline Major bleed events & Bivalirudin era June 2013-July 2014 & UFH era October 2014-May 2015 & $P$ value \\
\hline All bleeding events within 72 hours [\%] & $32[4.2]$ & $29[7.4]$ & 0.030 \\
\hline Hemoglobin drop [\%] & $28[4]$ & $25[6]$ & 0.040 \\
\hline Bleed at access site [\%] & $9[1]$ & $16[4]$ & 0.002 \\
\hline Retroperitoneal bleeding [\%] & 0 & $2[0.5]$ & 0.100 \\
\hline Gastrointestinal bleed [\%] & $1[0.1]$ & 0 & 1.000 \\
\hline Genitourinary bleed & 0 & 0 & - \\
\hline Major bleed event by medication use [\%] & $\mathrm{N}=32$ & $\mathrm{~N}=29$ & \\
\hline GPI & 12 [38] & 9 [31] & - \\
\hline
\end{tabular}

Hemoglobin drop is a decrease in hemoglobin more than $3 \mathrm{~g} / \mathrm{dL}$ within 72 hours. The lower part of the chart demonstrates primary anticoagulant and GPI bailout use with a major bleed during each era. Percentages are a function of the total number of bleeds in each era. NCDR, National Cardiovascular Data Registry; UFH, unfractionated heparin; GPI, glycoprotein Ilb/Illa inhibitor. 


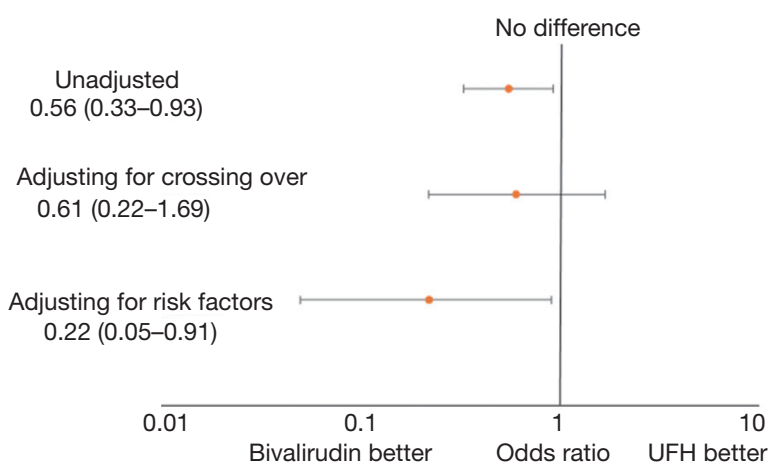

Figure 3 Bleeding odds with adjustment for risk factors. Graphical presentation of odds ratios/95\% CI of bleeding for patients in the bivalirudin era relative to the UFH era: unadjusted, adjusted for crossover, and adjusted for risk factors respectively. Adjustment for risk factors includes correction for catheterizations utilizing the opposite anticoagulant as well as GPI use, aspirin use, P2Y12 inhibitor use, age, sex, history of diabetes, CKD, access, STEMI presentation and cardiogenic shock. UFH, unfractionated heparin; GPI, glycoprotein IIb/IIIa inhibitor; CKD, chronic kidney disease; STEMI, ST elevation myocardial infarction.

events during the UFH era.

\section{Predictors of bleeding}

Age, sex, history of diabetes mellitus type 2, chronic kidney disease, femoral access, STEMI, and cardiogenic shock within 24 hours have previously been identified as risk factors for bleeding following PCI (11). Consistent with these results, we observed that STEMI presentation, GPI use, and cardiogenic shock were both highly associated with an increase in major bleeding, with odds ratios of 4.6 (95\% CI: 2.6-8.0), 14.5 (95\% CI: 7.8-26.8) and 21.1 (95\% CI: 8.0-55.3) respectively (Table 4). Femoral access (OR $=1.9,95 \%$ CI: $1.0-3.8)$ and smoking ( $\mathrm{OR}=1.8,95 \%$ CI: $1.03-3.0)$ were also marginally associated with an increase in major bleeding events.

Without adjustments, patients in the bivalirudin era had $44 \%$ lower odds of bleeding compared to the UFH era $(\mathrm{OR}=0.56,95 \%$ CI: 0.33-0.93) (Figure 3). After accounting for cases of UFH used as the primary anticoagulant during the bivalirudin era and bivalirudin used during the UFH era, the protective effect of bivalirudin attenuated $(\mathrm{OR}=0.61,95 \%$ CI: 0.22-1.69). After adjustment for factors associated with major bleeding after PCI (P2Y12 use, GPI use, age, sex, access site, STEMI, shock, diabetes mellitus
Table 4 Predictors of bleeding by multivariate analysis

\begin{tabular}{lr}
\hline Predictor & OR $(95 \% \mathrm{Cl})$ \\
\hline Age (per 5-year increment) & $1.0(0.90-1.1)$ \\
Female & $1.4(0.8-2.4)$ \\
Smoker & $1.8(1.03-3.0)$ \\
Hypertension & $0.9(0.4-1.7)$ \\
Diabetes & $1.5(0.9-2.6)$ \\
CKD stage 3 or worse & $1.1(0.6-1.9)$ \\
Myocardial infarction & $1.3(0.7-2.1)$ \\
STEMI & $4.6(2.6-8.0)$ \\
NSTEMI & $1.0(0.6-1.9)$ \\
Femoral access & $1.9(1.0-3.8)$ \\
Cardiogenic shock & $21.1(8.0-55.3)$ \\
Bivalirudin & $0.6(0.3-0.98)$ \\
UFH & $1.5(0.9-2.5)$ \\
GPI & $14.5(7.8-26.8)$ \\
\hline
\end{tabular}

This table demonstrates the odds of bleeding of with each factor present compared to when each factor is absent. Each odds ratio does not control for other risk factors for bleeding including whether bivalirudin or heparin was used. CKD, chronic kidney disease; STEMI, ST elevation myocardial infarction; NSTEMI, non-ST elevation myocardial infarction; UFH, unfractionated heparin; GPI, glycoprotein Ilb/Illa inhibitor.

and $\mathrm{CKD}$ ) bivalirudin was associated with $78 \%$ lower odds of bleeding (OR $=0.22$, 95\% CI: 0.05-0.91).

\section{Discussion}

This study demonstrates a modest, but statistically significant increase in bleeding when the primary anticoagulant was changed from bivalirudin to UFH, in an academic cardiac catheterization laboratory. The difference was primarily due to an increase in the number of patients with a hemoglobin drop after PCI without any overt signs of bleeding. There was no difference in vascular complications, need for transfusion or other overt bleeding events.

These data suggest that differences in bleeding events between UFH and bivalirudin may be minimized when radial access is used and when UFH is reserved for cases with low bleeding risk. A comparison of the bivalirudin era $v s$. UFH era in major bleeding events adjusting for crossover anticoagulant use did not show a significant difference in bleeding rates $(0.61,95 \%$ CI: $0.22-1.69)$. Only when our 
model adjusted for significant bleeding risk factors such as STEMI presentation, GPI use, cardiogenic shock, P2Y12 use, and access site utilized, were the differences between bivalirudin and heparin unmasked. It is important to note that the rate of transradial access was still relatively low $(\sim 35 \%)$ as compared to trials such as HEAT-PPCI and BRIGHT which may exaggerate the difference in bleeding outcomes between the two groups. Additionally, our survey of other risk factors for major bleeding events demonstrates that other variables such as STEMI presentation, cardiogenic shock, GPI use, femoral access, and smoking rank highly among factors that can increase the risk for bleeding events with this population, often with a greater measured effect than use of UFH. This suggests that the modest increased risk of bleeding seen with UFH use can be mitigated if patients in which it is used are carefully selected.

It is important to recognize that the vast majority of major bleeding events captured in this study were due to a hemoglobin drop of at least $3 \mathrm{~g} / \mathrm{dL}$ without any overt signs of clinical bleeding. The clinical significance of these bleeds is debatable. When analyzing only those bleeds that were clinically obvious, such as RP bleeds, and GI/GU bleeding, the rates of bleeding are very low and similar between the two groups. Therefore, the difference in bleeding rates between the two anticoagulation strategies is completely driven by the rates of hemoglobin drops and there is little data establishing clinical significance associated with these events.

We believe this study is strengthened by the systembased, rapid change in primary anticoagulation strategy, supported by all operators in our lab. This approach minimized the contribution of selection bias in the interpretation of these results and allows for a reasonable comparison of like populations in two closely related eras of treatment. The limitations of the study are similar to those of any single-center retrospective cross-sectional study with the primary limitation being generalizability. Another limitation to the study is the focus on only short-term bleeding outcomes instead of collecting data on longerterm efficacy outcomes. Studies such as the HEAT-PPCI, MATRIX and BRIGHT demonstrated conflicting results as to the difference in efficacy outcomes between bivalirudin monotherapy vs. UFH monotherapy. Supplementary data on efficacy outcomes from this study would have been helpful to contextualize the data from large multicenter randomized controlled trials. Also, this study was performed at an academic medical center with a relatively small number of patients. Performance of this study at an academic medical center did enable a rapid transition from bivalirudin to UFH. Despite these weaknesses, this single center approach offers a practical, realistic example of the conversion to a UFH-first strategy.

As cardiac catheterization labs analyze their use of anticoagulants for PCI, many factors, including cost, ease of use, and clinical outcomes, must be weighed in deciding what will be the preferred anticoagulant for PCI. This study provides a glimpse into the resultant outcomes of changing from a bivalirudin-first strategy to a UFH-first strategy in a large academic cardiac catheterization laboratory, in the context of other changes such as higher rates of radial access and diminishing uses of GPI. As the shift away from bivalirudin continues across the United States, a close evaluation of bleeding rates and outcomes is needed to ensure optimal care for patients undergoing PCI.

\section{Acknowledgements}

None.

\section{Footnote}

Conflicts of Interest: The authors have no conflicts of interest to declare.

Ethical Statement: This study was approved by the University of North Carolina Institutional Review Board (IRB 150573). IRB committee granted a waiver of informed consent given minimal risk to subjects/privacy, no risk to rights and welfare of subjects, impracticality of performance of study without waiver, and reasonable risk to privacy in relation to knowledge gained. Subjects were not contacted with pertinent information after conclusion as study aims to analyze the short-term outcomes resulting from protocol of past interventional procedures and would provide no direct harm or benefit to the subject at the time of analysis.

\section{References}

1. O'Gara PT, Kushner FG, Ascheim DD, et al. 2013 ACCF/ AHA guideline for the management of ST-elevation myocardial infarction: executive summary: a report of the American College of Cardiology Foundation/American Heart Association Task Force on Practice Guidelines: developed in collaboration with the American College of Emergency Physicians and Society for Cardiovascular Angiography and Interventions. Catheter Cardiovasc 
Interv 2013;82:E1-27.

2. Mehran R, Pocock SJ, Nikolsky E, et al. A risk score to predict bleeding in patients with acute coronary syndromes. J Am Coll Cardiol 2010;55:2556-66.

3. Feit F, Voeltz MD, Attubato MJ, et al. Predictors and impact of major hemorrhage on mortality following percutaneous coronary intervention from the REPLACE-2 Trial. Am J Cardiol 2007;100:1364-9.

4. Ndrepepa G, Berger PB, Mehilli J, et al. Periprocedural bleeding and 1-year outcome after percutaneous coronary interventions: appropriateness of including bleeding as a component of a quadruple end point. J Am Coll Cardiol 2008;51:690-7.

5. Lincoff AM, Bittl JA, Harrington RA, et al. Bivalirudin and provisional glycoprotein IIb/IIIa blockade compared with heparin and planned glycoprotein IIb/IIIa blockade during percutaneous coronary intervention: REPLACE-2 randomized trial. JAMA 2003;289:853-63.

6. Stone GW, Witzenbichler B, Guagliumi G, et al. Bivalirudin during primary PCI in acute myocardial infarction. N Engl J Med 2008;358:2218-30.

7. Stone GW, White HD, Ohman EM, et al. Bivalirudin in patients with acute coronary syndromes undergoing percutaneous coronary intervention: a subgroup analysis

Cite this article as: Jaswaney RV, Caughey MC, End C, Sudar P, Yeung M, Kaul P, Rossi JS, Stouffer GA, Vavalle JP. In-hospital outcomes after switching from a bivalirudinfirst strategy to an unfractionated heparin-first strategy for percutaneous coronary interventions. Cardiovasc Diagn Ther 2018;8(2):137-145. doi: 10.21037/cdt.2017.10.16 from the Acute Catheterization and Urgent Intervention Triage strategy (ACUITY) trial. Lancet 2007;369:907-19.

8. Jolly SS, Yusuf S, Cairns J, et al. Radial versus femoral access for coronary angiography and intervention in patients with acute coronary syndromes (RIVAL): a randomised, parallel group, multicentre trial. Lancet 2011;377:1409-20.

9. Shahzad A, Kemp I, Mars C, et al. Unfractionated heparin versus bivalirudin in primary percutaneous coronary intervention (HEAT-PPCI): an open-label, single centre, randomised controlled trial. Lancet 2014;384:1849-58.

10. Lee JA, Lee CR, Reed BN, et al. Implementation and evaluation of a CYP2C19 genotype-guided antiplatelet therapy algorithm in high-risk coronary artery disease patients. Pharmacogenomics 2015;16:303-13.

11. Rao SV, McCoy LA, Spertus JA, et al. An updated bleeding model to predict the risk of post-procedure bleeding among patients undergoing percutaneous coronary intervention: a report using an expanded bleeding definition from the National Cardiovascular Data Registry CathPCI Registry. JACC Cardiovasc Interv 2013;6:897-904. 